\title{
Intelligent Sensor Network for Vehicle Maintenance System
}

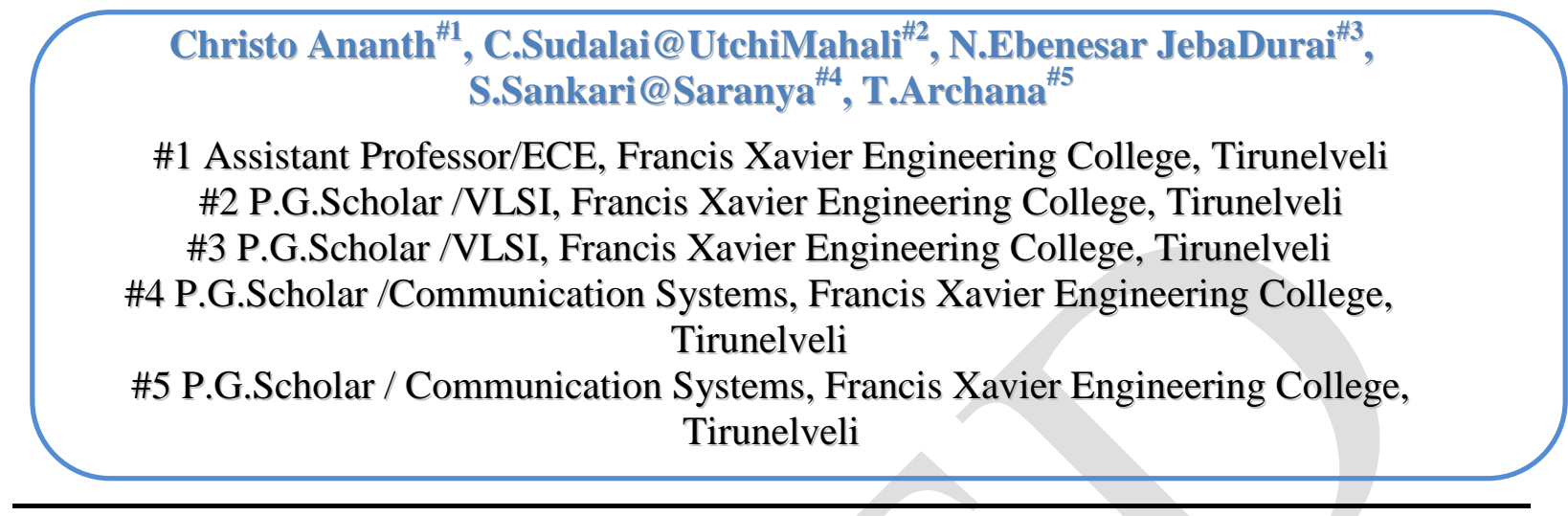

\section{ABSTRACT}

Modern automobiles are no longer mere mechanical devices; they are pervasively monitored through various sensor networks \& using integrated circuits and microprocessor based design and control techniques while this transformation has driven major advancements in efficiency and safety. In the existing system the stress was given on the safety of the vehicle, modification in the physical structure of the vehicle but the proposed system introduces essential concept in the field of automobile industry. It is an interfacing of the advanced technologies like Embedded Systems and the Automobile world. This "Intelligent Sensor Network for Vehicle Maintenance System" is best suitable for vehicle security as well as for vehicle's maintenance. Further it also supports advanced feature of GSM module interfacing. Through this concept in case of any emergency or accident the system will automatically sense and records the different parameters like LPG gas level, Engine Temperature, present speed and etc. so that at the time of investigation this parameters may play important role to find out the possible reasons of the accident. Further, in case of accident $\&$ in case of stealing of vehicle GSM module will send SMS to the Police, insurance company as well as to the family members.

Key words: Accident alert Module, Engine Temperature Control Module, Vibration Sensor, GSM Module

\section{Corresponding Author: Christo Ananth}

\section{INTRODUCTION}

Because of the high theft rate and various challenges coming from thief it is necessary to provide security from stealing vehicle or part of the vehicle. The first parameter is Engine temperature which sense the temperature of engine, according to which if the corresponding 
temperature is above the threshold temperature, then the action will be taken by microcontroller is to $\mathrm{ON}$ the cooling system for maintaining temperature of engine. The next parameter of the system is the servicing alert which gives you the alertness for the servicing of vehicle when the vehicle crosses the threshold kilometers whatever we have set. Next parameter is GSM Module. If the vehicle is steal or any accident case is occurred, and then by using this system it sends message to the family member or friend, Police \& Insurance Company, so that they are aware about the incidence. This will provide safety to the passenger as well as vehicle. Next is Speed control which is a important parameter because it is very important to maintain the speed in order to avoid accidents. The last parameter is to monitor the LPG level or any leakage of the gas in the vehicle.

MATERIALS AND METHODS

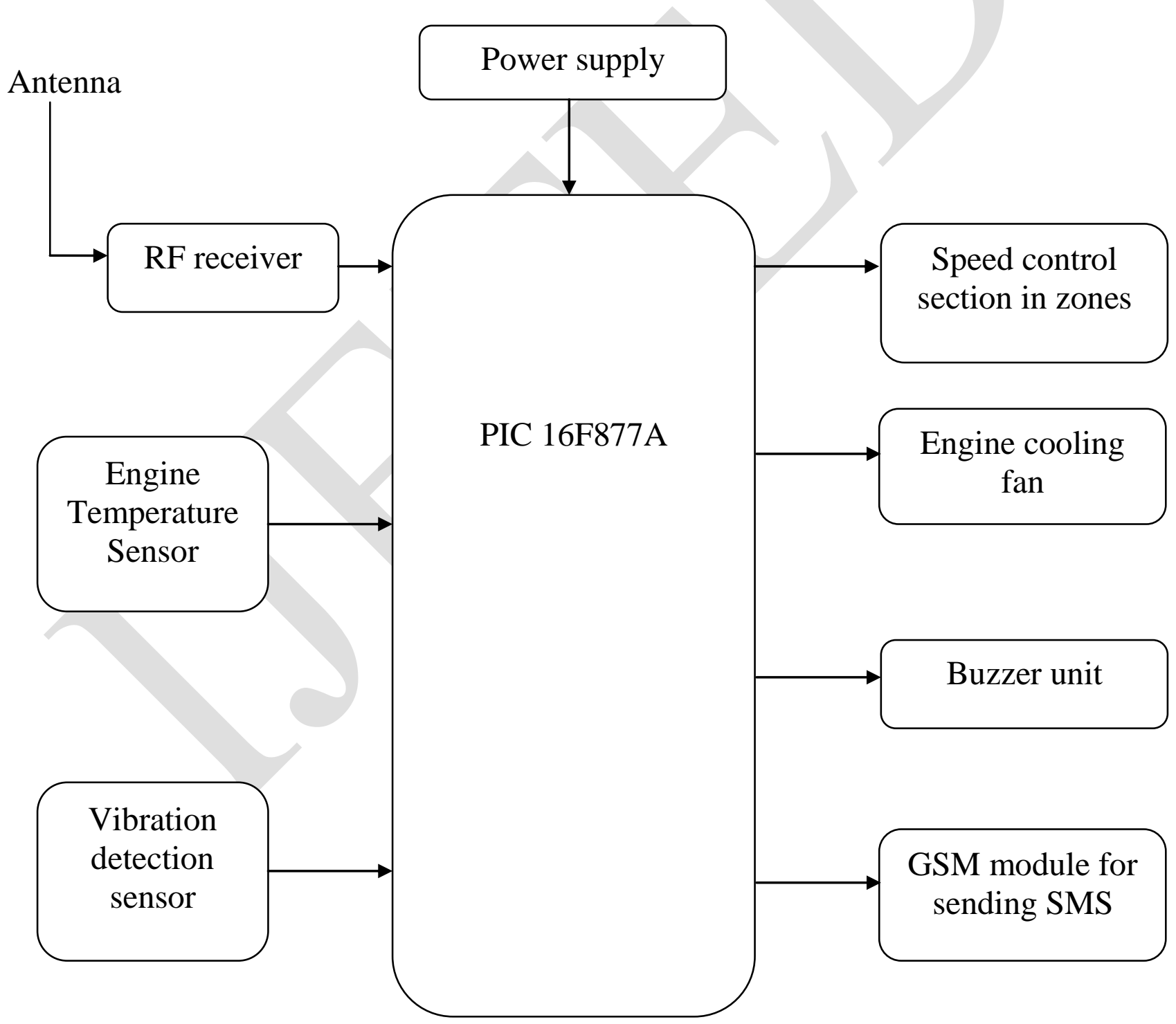

Fig 1: Block Diagram of Proposed System 
In Fig 1, Radio frequency Receiver receives the transmitted signals, amplifies it and provides the same to HT12D decoder IC. The decoder performs the inverse operation of the encoder, i.e., converting the serial bit stream back to the parallel form. The heart of this section is the PIC 16F877A micro-controller. It hosts major modules namely RF transmission/reception, Speed control section in different zones, Engine temperature monitoring system and Accident detection and intimation system

The ac voltage, typically $220 \mathrm{~V}$ rms, is connected to a transformer, which steps that ac voltage down to the level of the desired dc output. A diode rectifier then provides a fullwave rectified voltage that is initially filtered by a simple capacitor filter to produce a dc voltage. This resulting dc voltage usually has some ripple or ac voltage variation. A regulator circuit removes the ripples and also remains the same dc value even if the input dc voltage varies, or the load connected to the output dc voltage changes. This voltage regulation is usually obtained using one of the popular voltage regulator IC units.

The potential transformer will step down the power supply voltage $(0-230 \mathrm{~V})$ to $(0-6 \mathrm{~V})$ level. Then the secondary of the potential transformer will be connected to the precision rectifier, which is constructed with the help of op-amp. The advantages of using precision rectifier are it will give peak voltage output as DC, rest of the circuits will give only RMS output. Voltage regulators comprise a class of widely used ICs. Regulator IC units contain the circuitry for reference source, comparator amplifier, control device, and overload protection all in a single IC. IC units provide regulation of either a fixed positive voltage, a fixed negative voltage, or an adjustably set voltage. The regulators can be selected for operation with load currents from hundreds of mill amperes to tens of amperes, corresponding to power ratings from milli-watts to tens of watts.

A fixed three-terminal voltage regulator has an unregulated dc input voltage, $\mathrm{Vi}$, applied to one input terminal, a regulated dc output voltage, Vo, from a second terminal, with the third terminal connected to ground. The series 78 regulators provide fixed positive regulated voltages from 5 to 24 volts. Similarly, the series 79 regulators provide fixed negative regulated voltages from 5 to 24 volts.

\section{MICROCONTROLLER FEATURES}

The PIC Microcontroller has a host of features intended to maximize system reliability, minimize cost through elimination of external components, provide power saving operating modes and offer code protection. These are: OSC Selection, Reset, Power-on Reset (POR), and Power-up Timer (PWRT), Oscillator Start-up Timer (OST), Brown-out Reset (BOR), Interrupts, Watchdog Timer (WDT), SLEEP, Code protection, ID locations, In-Circuit Serial Programming, Low Voltage In-Circuit Serial Programming and In-Circuit Debugger

These devices have a watchdog timer, which can be shut off only through configuration bits. It runs off its own $\mathrm{RC}$ oscillator for added reliability. There are two timers that offer necessary delays on power-up. One is the Oscillator Start-up Timer (OST), intended to keep the chip in reset until the crystal oscillator is stable. The other is the Power-up Timer (PWRT), which provides a fixed delay of $72 \mathrm{~ms}$ (nominal) on power-up only. It is designed to keep the part in reset while the power supply stabilizes. With these two timers on-chip, most applications need no external reset circuitry. SLEEP mode is designed to offer a very low current powerdown mode. The user can wake-up from SLEEP through external reset, Watchdog Timer Wake- 
up, or through an interrupt. Several oscillator options are also made available to allow the part to fit the application. The RC oscillator option saves system cost while the LP crystal option saves power. A set of configuration bits are used to select various options.

A Power-on Reset pulse is generated on-chip when VDD rise is detected (in the range of $1.2 \mathrm{~V}-1.7 \mathrm{~V}$ ). To take advantage of the POR, tie the MCLR pin directly (or through a resistor) to VDD. This will eliminate external RC components usually needed to create a Power on Reset. A maximum rise time for VDD is specified. See Electrical Specifications for details. When the device starts normal operation (exits the reset condition), device operating parameters (voltage, frequency, temperature,...) must be met to ensure operation's these conditions are not met, the device must be held in reset until the operating conditions are met. Brown-out Reset may be used to meet the start-up conditions. For additional information, refer to Application Note, AN007, "Power-up Trouble Shooting" and (DS00007).

On power-up, the time-out sequence is as follows: The pWRT delay starts (if enabled) when a POR reset occurs. Then OST starts counting 1024 oscillator cycles when PWRT ends (LP, XT, and HS). When the OST ends, the device comes out of RESET. If MCLR is kept low long enough, the time-outs will expire. Bringing MCLR high will begin execution immediately. This is useful for testing purposes or to synchronize more than one PIC16CXX device operating in parallel. Table 12-5 shows the reset conditions for the STATUS, PCON and PC registers, while Table 12-6 shows the reset conditions for all the registers.

The Power Control/Status Register, PCON, has up to two bits depending upon the device. Bit0 is Brown-out Reset Status bit; BOR. Bit BOR is unknown on a Power-on Reset. It must then be set by the user and checked on subsequent resets to see if bit BOR cleared, indicating a BOR occurred. The BOR bit is a "don't care" bit and is not necessarily predictable if the Brownout Reset circuitry is disabled (by clearing bit BODEN in the Configuration Word). Bit1 is POR (Power-on Reset Status bit). It is cleared on a Power-on Reset and unaffected otherwise. The user must set this bit following a Power-on Reset.

The Watchdog Timer is as a free running on-chip $\mathrm{RC}$ oscillator which does not require any external components. This $\mathrm{RC}$ oscillator is separate from the $\mathrm{RC}$ oscillator of the OSC1/CLKIN pin. That means that the WDT will run, even if the clock on the OSC1/CLKIN and OSC2/CLKOUT pins of the device has been stopped, for example, by execution of a SLEEP instruction. During normal operation, a WDT time-out generates a device RESET (Watchdog Timer Reset). If the device is in SLEEP mode, a WDT time-out causes the device to wake-up and continue with normal operation (Watchdog Timer Wake-up). The TO bit in the STATUS register will be cleared upon a Watchdog Timer time-out.

\section{SPEED CONTROL SECTION IN DIFFERENT ZONES}

Pulse-width modulation (PWM), or pulse-duration modulation (PDM), is a commonly used technique for controlling power to inertial electrical devices, made practical by modern electronic power switches. The average value of voltage (and current) fed to the load is controlled by turning the switch between supply and load on and off at a fast pace. The longer the switch is on compared to the off periods, the higher the power supplied to the load is. An electric motor is an electromechanical device that converts electrical energy into mechanical energy. Most electric motors operate through the interaction of magnetic fields and currentcarrying conductors to generate force. The reverse process, producing electrical energy from mechanical energy, is done by generators such as an alternator or a dynamo; some electric 
motors can also be used as generators, for example, a traction motor on a vehicle may perform both tasks. Electric motors and generators are commonly referred to as electric machines.

Medium-size motors of highly standardized dimensions and characteristics provide convenient mechanical power for industrial uses. The very largest electric motors are used for propulsion of ships, pipeline compressors, and water pumps with ratings in the millions of watts. Electric motors may be classified by the source of electric power, by their internal construction, by their application, or by the type of motion they give. Fig. 2 shows the Speed Control Section in different
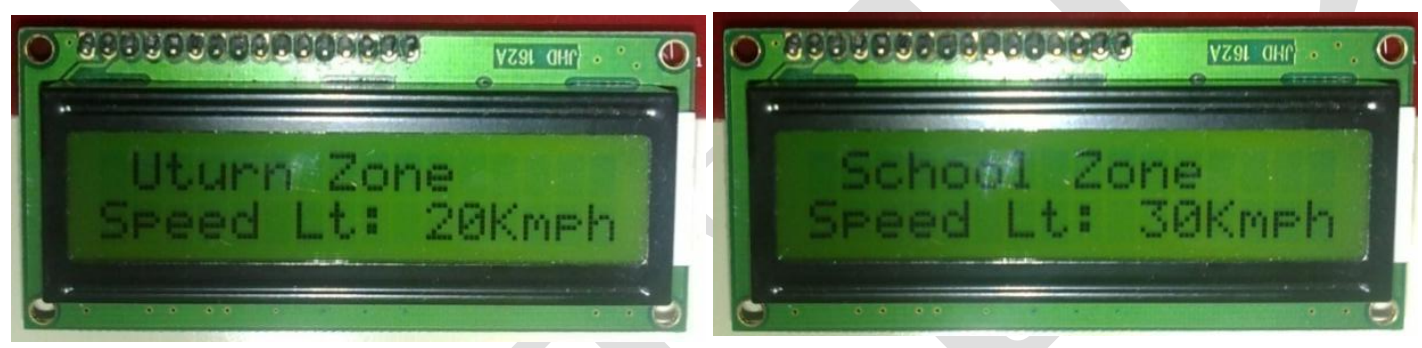

Fig 2: Speed Control section in different zones

\section{ACCIDENT ALERT SECTION}

In the modern traffic the vehicle accidents are becoming common and unavoidable. But it causes severe trauma to the human lives, risking their chance of survival. Hence sensing the occurrence of the accident and informing it to the ambulance service or to a known friend increases the chance of survival. This module incorporates a vibration sensor to sense the excessive vibrations due to the accident and a GSM module to alert a local ambulance service or a person via a SMS. The two main components in this module are a vibration sensor and a GSM module.

A piezoelectric vibration sensor is a device that uses the piezoelectric effect to measure pressure, acceleration, strain or force by converting them to an electrical charge. GSM/GPRS TTL UART Modem is built with Dual Band GSM/GPRS engine- SIM900, works on frequencies $900 / 1800 \mathrm{MHz}$. The Modem is coming with selectable interfacing voltage, which allows you to connect $5 \mathrm{~V} \& 3 \mathrm{~V} 3$ microcontroller directly without any level conversion chips. The baud rate is configurable from 9600-115200 through AT command. 


\section{ENGINE TEMPERATURE CONTROL SECTION}

This system proposes a method to maintain the engine temperature by employing a temperature sensor and a cooling fan. The temperature sensor periodically measures the current engine temperature and sends the value to the microcontroller. The cooling fan is used to blow the air over the surface of the engine to reduce the temperature. Thus maintaining the temperature of the engine provides a better performance. LM35 is a precision IC temperature sensor with its output proportional to the temperature (in ${ }^{\circ} \mathrm{C}$ ). The sensor circuitry is sealed and therefore it is not subjected to oxidation and other processes. With LM35, temperature can be measured more accurately than with a thermistor. It also possess low self heating and does not cause more than $0.1{ }^{\circ} \mathrm{C}$ temperature rise in still air. The operating temperature range is from $55^{\circ} \mathrm{C}$ to $150^{\circ} \mathrm{C}$. The output voltage varies by $10 \mathrm{mV}$ in response to every ${ }^{\circ} \mathrm{C}$ rise/fall in ambient temperature, i.e., its scale factor is $0.01 \mathrm{~V} /{ }^{\circ} \mathrm{C}$.

\section{RESULTS AND DISCUSSION}

\section{SPEED CONTROL SECTION IN DIFFERENT ZONES}

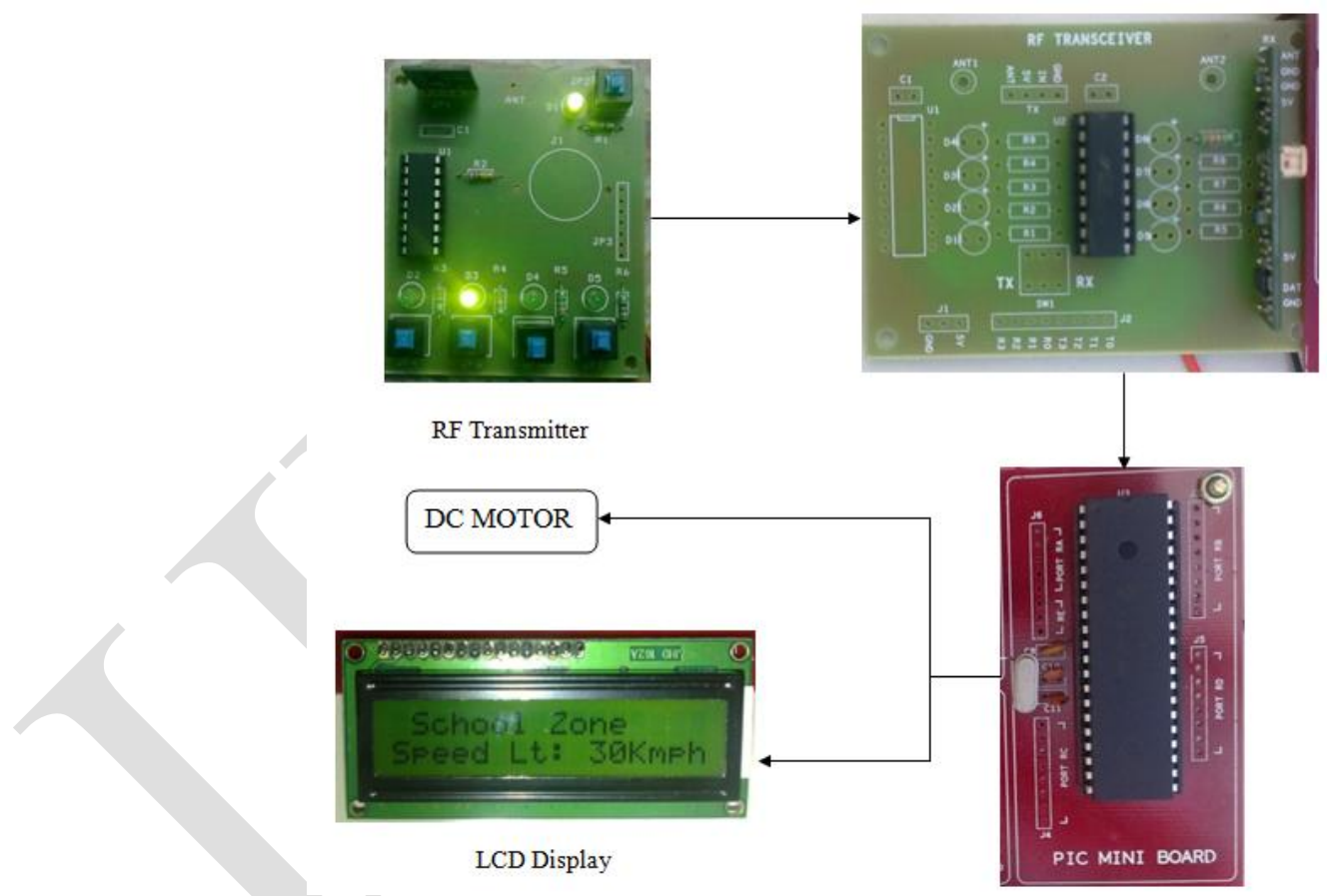

Fig 3: Speed Control section in School zone

Fig.3. shows the signal flow between the modules and the selection of school zone. The $\mathrm{RF}$ unit is placed at port $\mathrm{B}$ of the microcontroller from pins $\mathrm{RB} 0$ to $\mathrm{RB} 3$. 


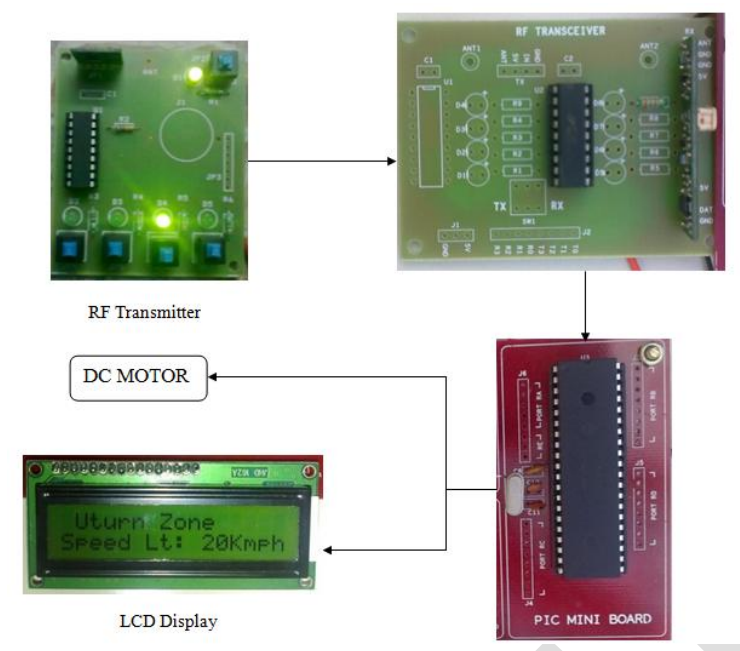

Fig 4: Speed Control section in U-turn zone

Fig.4. shows the signal flow between the modules and the selection of u-turn zone.

\section{ENGINE TEMPERATURE CONTROL SECTION}

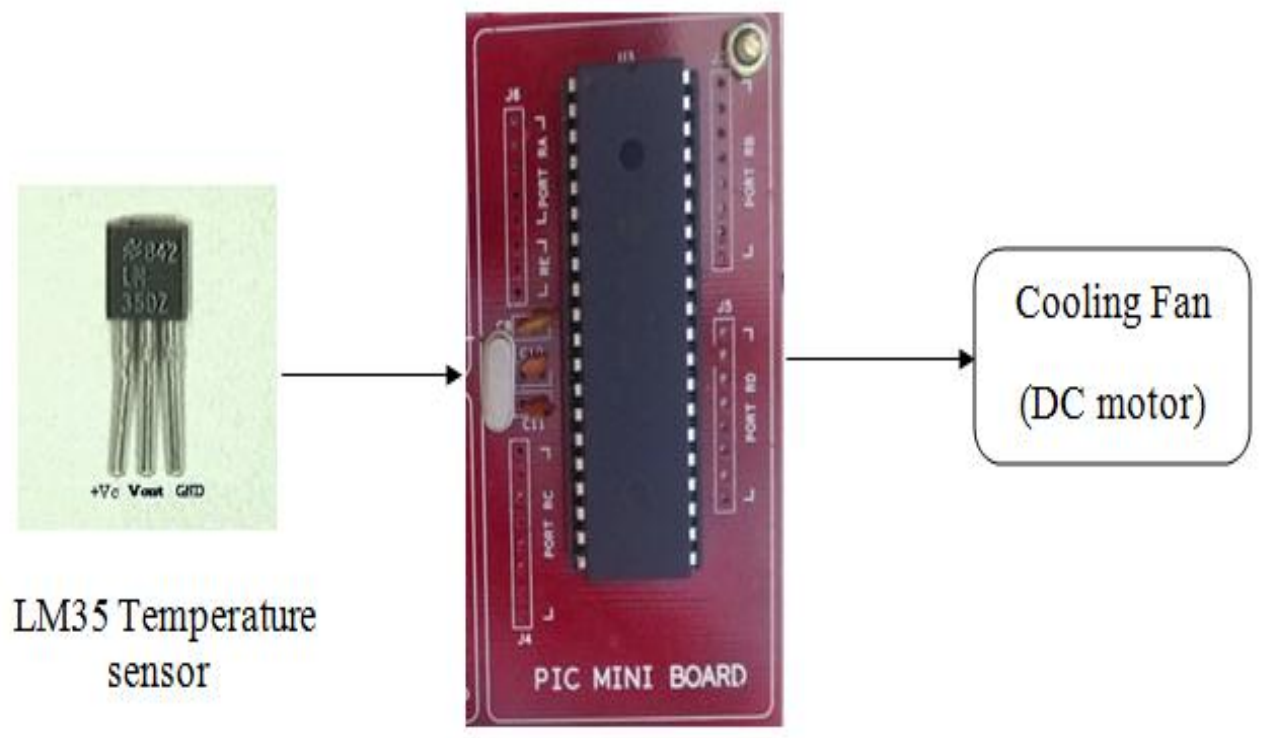

Fig 5: Engine Temperature Control Section

Fig.5. shows the engine Temperature Control Section. The LM35 temperature sensor is connected to RA0 pin of the microcontroller. It senses the temperature and sends the voltage signal to the microcontroller. The microcontroller activates the cooling fan. After the engine is cooled below the threshold temperature, the cooling fan is switched off. 


\section{ACCIDENT ALERT SECTION}

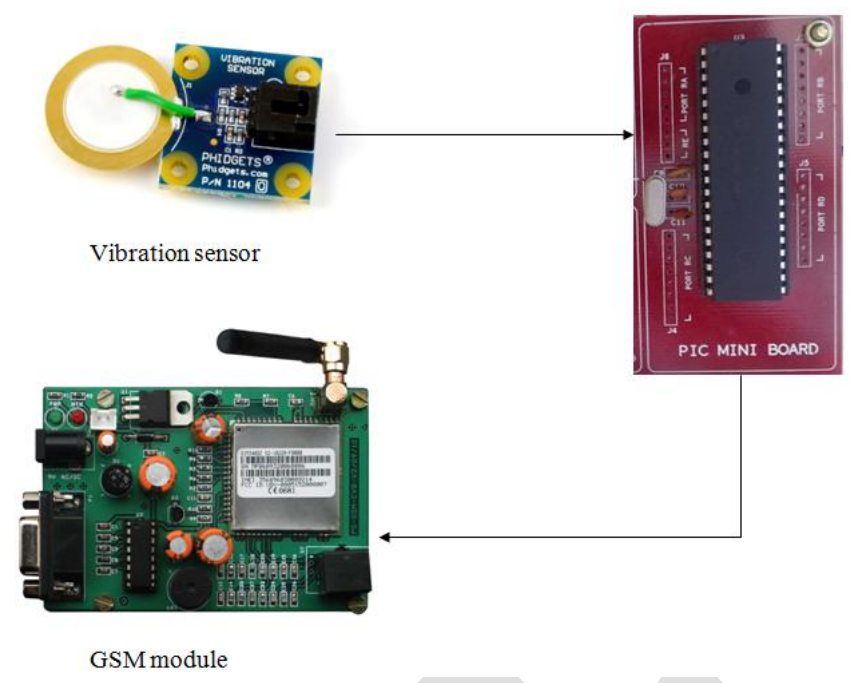

Fig 6: Accident Alert Section

The vibration sensor detects the excessive vibrations caused due to the occurrence of accident. It is connected to the pin RA1. The microcontroller sends an alert sms to the predefined number defined in the programming part via GSM module.

\section{CONCLUSION}

Modern automobiles are no longer mere mechanical devices; they are pervasively monitored through various sensor networks \& using integrated circuits and microprocessor based design and control techniques while this transformation has driven major advancements in efficiency and safety. In the existing system the stress was given on the safety of the vehicle, modification in the physical structure of the vehicle but the proposed system introduces essential concept in the field of automobile industry. It is an interfacing of the advanced technologies like Embedded Systems and the Automobile world. This "Intelligent Sensor Network for Vehicle Maintenance System" is best suitable for vehicle security as well as for vehicle's maintenance. Further it also supports advanced feature of GSM module interfacing. Through this concept in case of any emergency or accident the system will automatically sense and records the different parameters like LPG gas level, Engine Temperature, present speed and etc. so that at the time of investigation this parameters may play important role to find out the possible reasons of the accident. Further, in case of accident \& in case of stealing of vehicle GSM module will send SMS to the Police, insurance company as well as to the family members.

\section{REFERENCES}

1. Huaqun Guo, H.S. Cheng, Y.D. Wu, "An Automotive Security System for Anti-theft “, Eighth International Conference on Networks, vol.9, issue 3,pp.421-426,2012 
2. Lien-Wu Chen, Kun-Ze Syue, and Yu-Chee Tseng "A Vehicular Surveillance and Sensing System for Security Applications", Department of Computer Science, National Chiao-Tung University, Hsin-Chu, 300, Taiwan,2011

3. V.Kaul, Daniel J.S.Lim "Methods and Devices to Communicate via SMS After a Security Intrusion ", $1^{\text {st }}$ International Conference on Sensing Technology, PalmerstonNorth-NewZealand, November 21-23,vol 4,issue 5,pp 664-668,2010

4. K.Liu, Chen Chan Yang "Of Moore \& Gene" $22^{\text {nd }}$ International Conference on VLSI design, $8^{\text {th }}$ International Conference on Embedded System, $5^{\text {th }}-9^{\text {th }}$ January, Delhi,2009

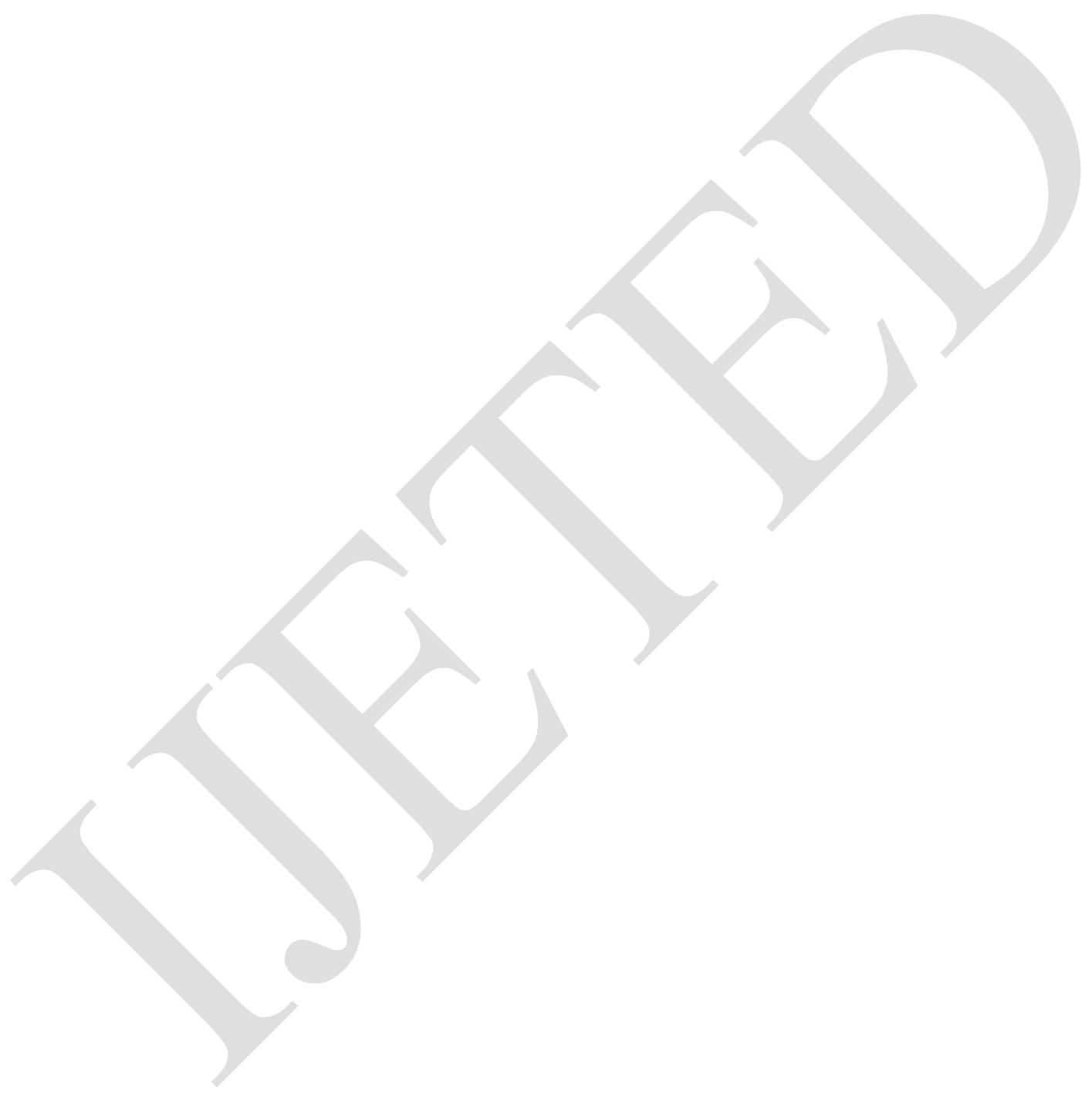

